\title{
Electrochemical Sensor for the Detection of 1-Hydroxypyrene Based on Composites of PAMAM-Regulated Chromium-Centered Metal-Organic Framework Nanoparticles and Graphene Oxide
}

Hong Cui ${ }^{\text {a, } \dagger, *}$, Shuaishuai Cui ${ }^{\text {a, }}$, Qiuju Tian ${ }^{\text {a }}$, Siyuan Zhang ${ }^{\text {a }}$, Mingxiu Wang ${ }^{\text {a }}$, Ping Zhang ${ }^{\text {a }}$, Yunfeng Liu ${ }^{\text {a }}$, Jialing Zhang ${ }^{\text {a }}$, Xiangjun $\mathrm{Li}^{\text {b,* }}$

${ }^{a}$ School of Public Health, Shanxi Medical University, 56Xinjian South Road, Taiyuan, 030001, China

${ }^{\mathrm{b}}$ School of Chemical Sciences, University of Chinese Academy of Sciences, $19 \mathrm{~A}$ Yuquan Road, Beijing, 100049, China

\footnotetext{
* Corresponding authors. E-mail addresses: cuihong19872007@126.com (H. Cui), lixiangj@ucas.ac.cn (X. Li).

$\dagger$ co-first authors
} 


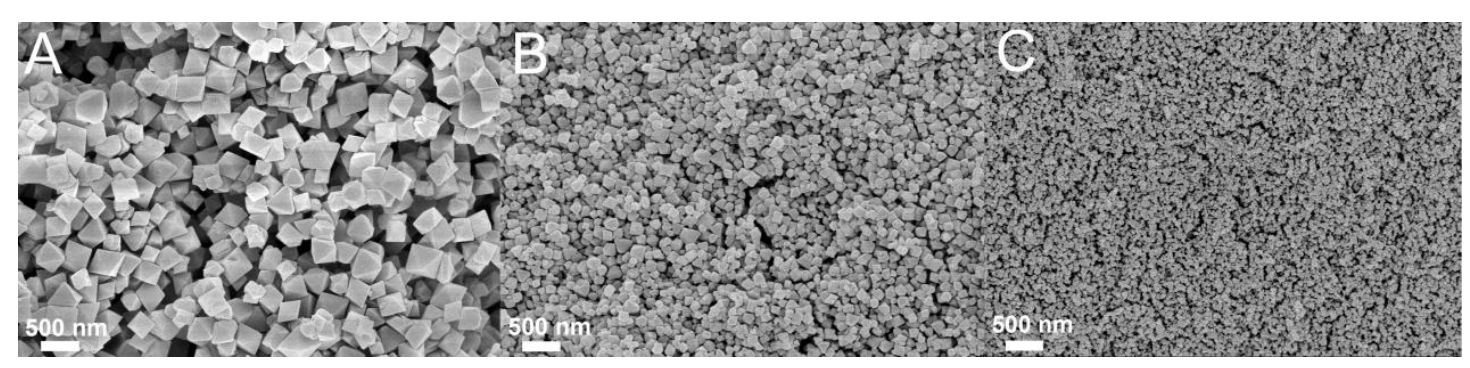

Figure S1. SEM images of PAMAM/Cr-MOF after the addition of: (A) 25, (B) 50 and (C) $75 \mu \mathrm{L}$ of PAMAM.

A

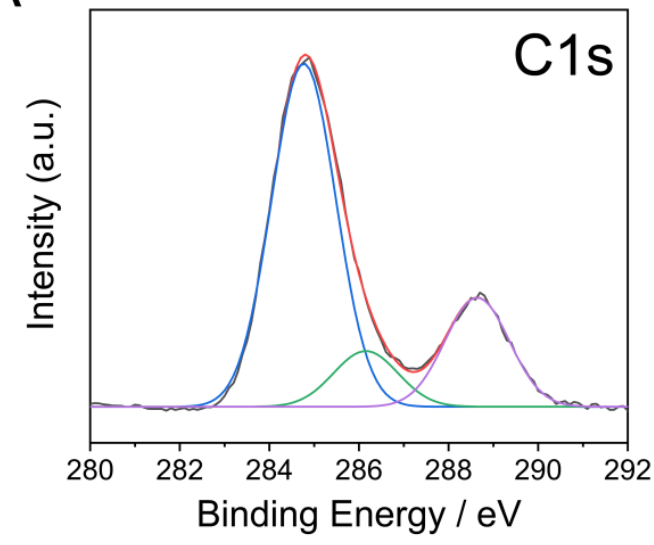

C

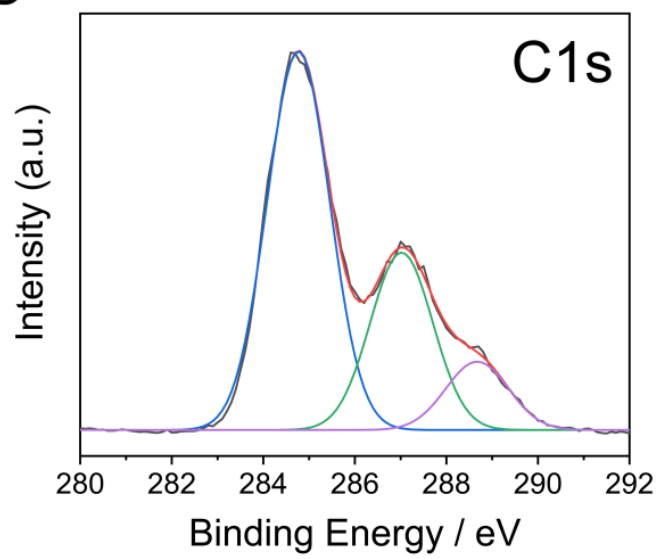

B

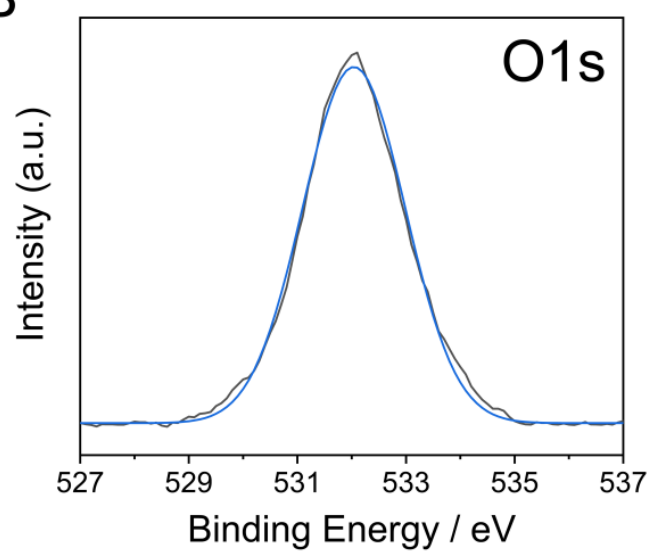

D

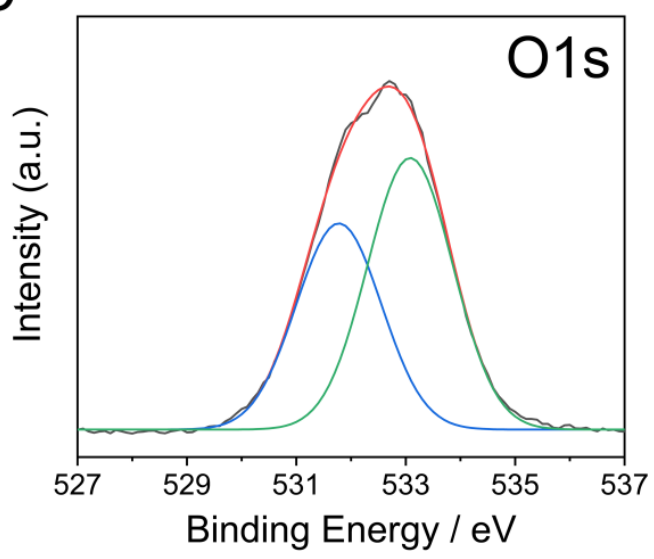


Figure S2. XPS spectrum of the C 1s (A) and O 1s (B) peak of PAMAM/Cr-MOF; the $\mathrm{C}$ 1s $(\mathrm{C})$ and $\mathrm{O}$ 1s (D) peak of PAMAM/Cr-MOF/GO.

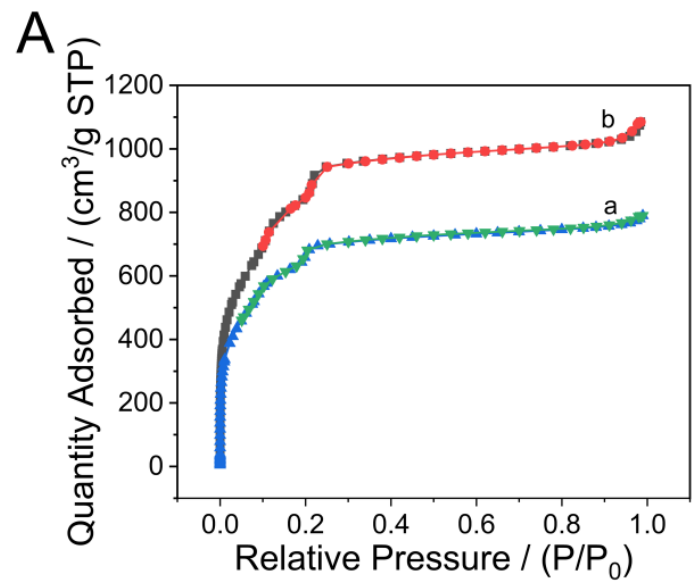

B

Figure S3. (A) $\quad \mathrm{N}_{2}$ adsorption-desorption isotherm of $\mathrm{Cr}-\mathrm{MOF}$ (a) and PAMAM/Cr-MOF (b). (B) TGA graphs of GO (a), PAMAMA/Cr-MOF (b), and PAMAM/Cr-MOF/GO (c).

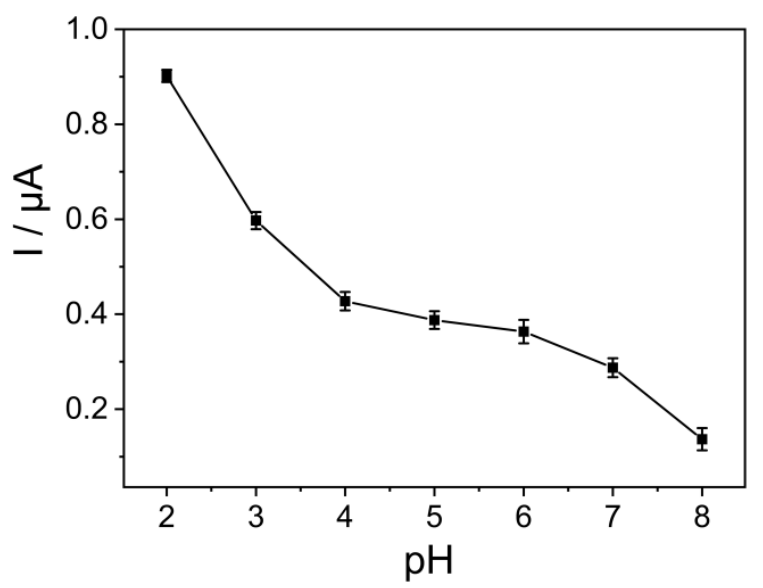

Figure S4. The relationship between the peak currents and $\mathrm{pH}$ values of the supporting electrolyte. 

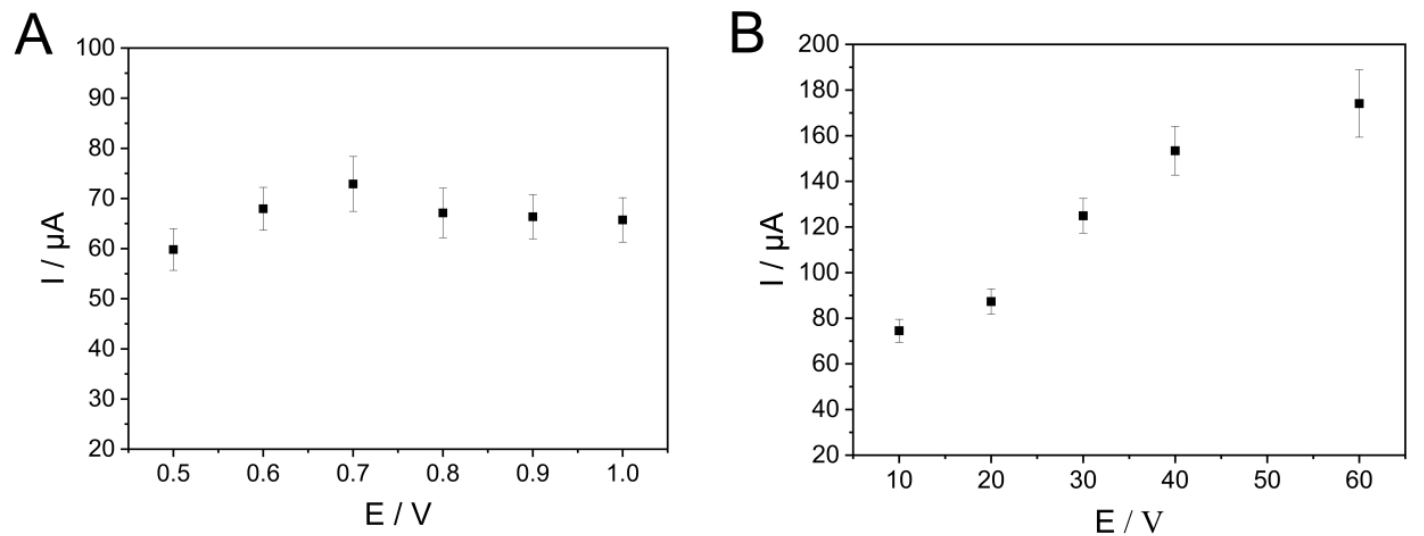

Figure S5. The relationships between the peak currents and the (A) deposition potential and (B) deposition time.

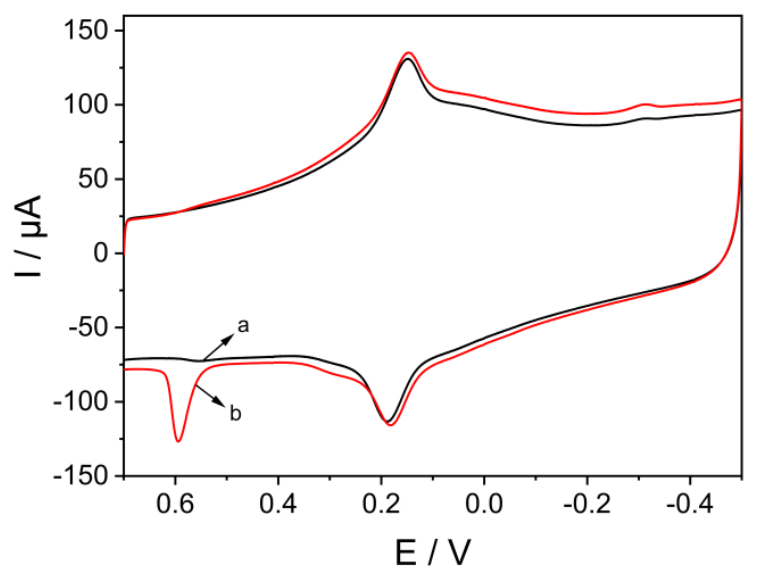

Figure S6. CVs of $5 \mu \mathrm{M}$ 1-OHPyr in the absence (a) and presence (b) of $500 \mu \mathrm{M}$ UA at PAMAM/Cr-MOF/ERGO/GCE in $0.05 \mathrm{M}$ PBS (pH 2.0) containing $0.2 \mathrm{M} \mathrm{NaCl}$. Scan rate: $100 \mathrm{mV} \mathrm{s}^{-1}$. 